\title{
The Extended Kalman Filter for Nonlinear State Estimation in a U-loop Bioreactor
}

Ritschel, Tobias K. S.; Boiroux, Dimitri; Nielsen, Marcus Krogh; Huusom, Jakob Kjøbsted; Jørgensen, Sten Bay; Jørgensen, John Bagterp

Published in:

2019 IEEE Conference on Control Technology and Applications (CCTA)

Link to article, DOI:

10.1109/ccta.2019.8920643

Publication date:

2019

Document Version

Peer reviewed version

Link back to DTU Orbit

Citation (APA):

Ritschel, T. K. S., Boiroux, D., Nielsen, M. K., Huusom, J. K., Jørgensen, S. B., \& Jørgensen, J. B. (2019). The Extended Kalman Filter for Nonlinear State Estimation in a Ü-loop Bioreactor. In 2019 IEEE Conference on Control Technology and Applications (CCTA) (pp. 920-925). IEEE. https://doi.org/10.1109/ccta.2019.8920643

\section{General rights}

Copyright and moral rights for the publications made accessible in the public portal are retained by the authors and/or other copyright owners and it is a condition of accessing publications that users recognise and abide by the legal requirements associated with these rights.

- Users may download and print one copy of any publication from the public portal for the purpose of private study or research.

- You may not further distribute the material or use it for any profit-making activity or commercial gain

- You may freely distribute the URL identifying the publication in the public portal 


\title{
The Extended Kalman Filter for Nonlinear State Estimation in a U-loop Bioreactor
}

\author{
Tobias K. S. Ritschel, Dimitri Boiroux, Marcus Krogh Nielsen, Jakob Kjøbsted Huusom, \\ Sten Bay Jørgensen, John Bagterp Jørgensen
}

\begin{abstract}
In this paper, we consider nonlinear state estimation in the U-loop reactor for single-cell protein (SCP) production. The model of the U-loop reactor is a mixture of stochastic partial differential equations and stochastic differential equations which are stiff. By a typical finite-volume spatial discretization, the resulting system of stochastic differential equations for numerical simulation and state estimation has 83 states. We investigate and discuss the continuous-discrete EKF for state estimation in this high-dimensional and stiff continuous-discrete-time system.
\end{abstract}

\section{INTRODUCTION}

State estimation as a filter algorithm is a central component in monitoring, fault detection, and model predictive control. When the process is nonlinear, a nonlinear filtering algorithm is needed. The nonlinear filtering problem is a computationally hard problem. It is well known that the solution of the Fokker-Planck equation (Kolmogorov's forward equation), i.e. the exact probability density function of the states, is the optimal solution to the state estimation problem [1]. However, due to the curse of dimensionality and computational tractability, the Fokker-Planck equation is restricted to low dimensional problems and cannot be applied to most practical problems. It can certainly not be applied to estimation in systems where the model is a number of partial and ordinary differential equations. Therefore, a number of alternative approximate nonlinear filtering algorithms are used. These algorithms are also challenged by the high state dimensionality of models arising from finite-volume discretizaton of partial differential equation systems. Singlecell protein $(\mathrm{SCP})$ production in the U-loop reactor studied in this paper gives rise to a coupled system of partial and ordinary differential equations. Consequently, a finite-volume spatial discretization gives rise to a high-dimensional system. In this paper, we investigate the extended Kalman filter $(\mathrm{EKF})$ for nonlinear state estimation in this system.

\footnotetext{
*This project has received funding from the European Union's Horizon 2020 research and innovation programme under grant agreement No 723661, Spire project: "Coordinating Optimisation of Complex Industrial Processes" (www.cocop-spire.eu). This article reflects only the author's views and the Commission is not responsible for any use that may be made of the information contained therein. This work also received funding in the project EUDP 64013-0558 in the IEA annex for energy efficient process control. Tobias K. S. Ritschel, Dimitri Boiroux, Marcus Krogh Nielsen, and John Bagterp Jørgensen are with the Department of Applied Mathematics and Computer Science, Technical University of Denmark, DK-2800 Kgs. Lyngby, Denmark. Jakob Kjøbsted Huusom and Sten Bay Jørgensen are with the Department of Chemical and Biochemical Engineering, Technical University of Denmark, DK-2800 Kgs. Lyngby, Denmark. Tobias K. S. Ritschel is also with 2-control ApS, DK-2900 Hellerup, Denmark. jbjo@dtu. dk
}

\section{A. Nonlinear filtering algorithms}

The Kalman filter is an optimal filter for linear systems with normally distributed process and measurement noise [2]. When the system is nonlinear, high-dimensional, and evolving in continuous-time, a number of different approximations such as the EKF and the unscented Kalman filter (UKF) have been suggested, while approaches based on the Fokker-Planck equation, hidden Markov models, particle filtering, or the ensemble Kalman filter (EnKF) suffer from the curse of dimensionality [1]-[7]. Most literature on nonlinear filtering algorithms for process applications considers low dimensional and discrete-discrete systems with only few details related to the numerical methods [8]-[13]. In this paper, we formulate the EKF for a high-dimensional continuous-discrete system stemming from a mixed system of stochastic partial and ordinary differential equations that are measured at discrete times. Such systems are ubiquitous in process systems engineering. We use the EKF because it is computationally efficient and involves few tuning parameters, e.g. compared to the UKF. Furthermore, we demonstrate the relevance of the formulated EKF by application to a novel process from industrial biotechnology.

\section{B. Single cell protein production in a U-loop reactor}

Methanotrophs can grow on cheap carbon sources such as methane or methanol. They have a high protein content and can be used to produce SCP. SCP can be used for animal feed and thereby sustain a growing human population. SCP may be produced using a U-loop reactor. However, the operation of such a reactor and in particular the startup is non-trivial. Using a mathematical model that describes the dynamics of SCP production in a U-loop reactor [14]-[18], we use the EKF to estimate the state of the reactor during startup based on the economic optimizing control strategy described by Drejer et al. [19]. The information provided by this state estimation is central to economic nonlinear model predictive control [20] for economically optimal operation and startup of the U-loop reactor. Previously, Olsen et al. [21] computed the optimal steady-state operating points, but did not consider the startup. Furthermore, state estimation has not previously been considered for the U-loop reactor. The computation and implementation of the optimal startup profile is challenging as the optimal profile turns out to be an unstable attractor, i.e. an uncontrolled system diverges from this optimal trajectory. Thus, using an open-loop control strategy would cause the startup of the U-loop reactor to fail [19]. 


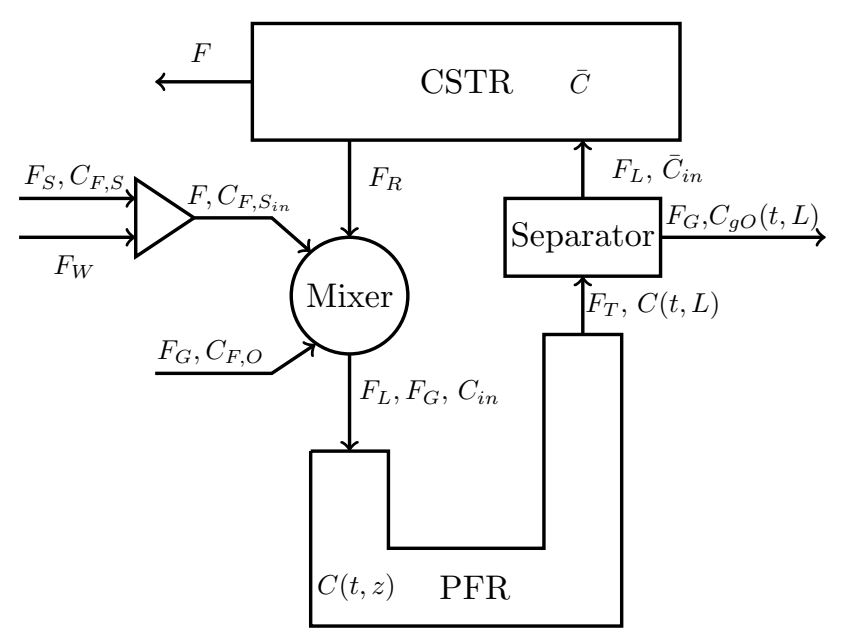

Fig. 1. U-loop reactor diagram for mathematical modeling.

\section{Paper organization}

The remaining part of the paper is organized as follows. Section II presents the mathematical model for SCP production in a U-loop reactor with methanol as the feed. Section III discusses the EKF for continuous-discrete systems, and in Section IV, we present the numerical example of state estimation during startup of the U-loop reactor. Section V summarizes the conclusions.

\section{MATHEMATICAL MODEL}

Fig. 1 is a conceptual diagram used to derive a mathematical model of SCP production in the U-loop reactor [14], [15], [19], [21]. At its inlet, the U-loop section consists of a mixer, in which the recirculated mixture from the top tank is mixed with feed substrate (methanol), feed water, and feed gas (oxygen). The remaining part of the U-loop is modeled as a liquid-gas phase plug flow reactor (PFR). The end of the U-loop is modeled as an ideal separator that completely separates the gas phase from the liquid phase. The top tank is modeled as a liquid phase continuous stirred tank reactor (CSTR). A liquid mixture of water, biomass, substrate, and dissolved gas is harvested from the top tank. The top tank also recirculates part of the mixture.

\section{A. Mixing section}

The gas flow rate at the inlet of the PFR is equal to the feed gas flow rate, $F_{G}$. The liquid flow rate, $F_{L}$, to the inlet of the PFR is obtained by a total static mass balance:

$$
F_{L}=F_{R}+F_{S}+F_{W}
$$

$F_{R}$ is the flow rate of liquid from the top tank to the U-loop, $F_{S}$ is the flow rate of feed substrate, and $F_{W}$ is the flow rate of feed water. Component mass balances for the biomass, $X$, the substrate, $S$, the oxygen dissolved in the liquid phase, $O$, and the oxygen in the gas phase, $g O$, give the following inlet concentrations to the PFR

$$
\begin{aligned}
C_{i n, X} & =\frac{F_{R} \bar{C}_{X}}{F_{L}}, \\
C_{i n, S} & =\frac{F_{S} C_{F, S}+F_{R} \bar{C}_{S}}{F_{L}}, \\
C_{i n, O} & =\frac{F_{R} \bar{C}_{O}}{F_{L}}, \\
C_{i n, g O} & =C_{F, O} .
\end{aligned}
$$

$\bar{C}_{X}, \bar{C}_{S}$, and $\bar{C}_{O}$ denote the concentrations of biomass $(X)$, substrate $(S)$, and oxygen $(O)$ in the top tank.

\section{B. The U-loop modeled as a plug-flow reactor}

The corresponding inlet fluxes, $N_{i}$, to the PFR are

$$
N_{i}(t, 0)=v C_{i n, i}(t), \quad i \in\{X, S, O, g O\},
$$

where the linear velocity is computed as

$$
v=\frac{F_{L}+F_{G}}{A} .
$$

$A$ is the cross-sectional area of the U-loop pipe. The concentrations, $C_{i}=C_{i}(t, z)$, of all components, $i \in$ $\{X, S, O, g O\}$, are computed as functions of time, $t$, and position, $z$, by the following mass balances

$$
\begin{aligned}
\frac{\partial C_{X}}{\partial t} & =-\frac{\partial N_{X}}{\partial z}+R_{X} \\
\frac{\partial C_{S}}{\partial t} & =-\frac{\partial N_{S}}{\partial z}+R_{S} \\
\frac{\partial C_{O}}{\partial t} & =-\frac{\partial N_{O}}{\partial z}+R_{O}+\frac{1}{1-\varepsilon} J_{g l, O}, \\
\frac{\partial C_{g O}}{\partial t} & =-\frac{\partial N_{g O}}{\partial z}-\frac{1}{\varepsilon} J_{g l, O},
\end{aligned}
$$

for $t_{a} \leq t \leq t_{b}$ and $0 \leq z \leq L$. $t_{a}$ is the initial time, $t_{b}$ is the final time, and $L$ is the length of the U-loop pipe. $N_{i}=$ $N_{i}\left(C_{i}(t, z)\right)=N_{i}(t, z)$ denotes the flux of each component, $i \in\{X, S, O, g O\}, R_{i}=R_{i}(t, z)$ denotes the production rate for $i \in\{X, S, O\}$, and $J_{g l, O}=J_{g l, O}(t, z)$ is the transfer rate of oxygen from the gas phase to the liquid phase. $\varepsilon=$ $F_{G} /\left(F_{G}+F_{L}\right)$ is the fraction of gas in each cross section. It is assumed to be constant throughout the U-loop pipe.

Danckwerts' conditions describe the outlet boundary conditions of the PFR, i.e.

$$
\frac{\partial C_{i}}{\partial z}(t, L)=0, \quad i \in\{X, S, O, g O\}, \quad t_{a} \leq t \leq t_{b} .
$$

\section{Gas-liquid separating section}

We assume that the gas and liquid are perfectly and instantaneously separated when the fast flowing gas-liquid mixture from the U-loop pipe enters the top tank. This is modeled as an ideal static gas-liquid separator, where the gas is completely removed, and the liquid phase enters the liquid phase in the top tank. This implies that the inlet concentrations of the liquid phase to the top tank are

$$
\bar{C}_{i n, i}(t)=C_{i}(t, L), \quad i \in\{X, S, O\}, \quad t_{a} \leq t \leq t_{b} .
$$




\section{Top-tank model}

The top tank is modeled as a CSTR with a constant liquid volume. The mass balances for the components in the liquid phase are

$\frac{d \bar{C}_{i}}{d t}=\bar{D}\left(\bar{C}_{i n, i}-\bar{C}_{i}\right)+R_{i}\left(\bar{C}_{X}, \bar{C}_{S}, \bar{C}_{O}\right), i \in\{X, S, O\}$,

for $t_{a} \leq t \leq t_{b}$. The dilution rate is $\bar{D}=\bar{F} / V$, where $\bar{F}=F_{L}$ and $V$ is the liquid volume in the top tank. As the liquid volume in the top tank is constant, the flow rate of the product stream from the top tank is

$$
F=\bar{F}-F_{R}=F_{L}-F_{R}=F_{S}+F_{W} .
$$

\section{E. Convective and diffusive transport}

The flux, $N_{i}$, consists of convective transport, $v C_{i}$, and diffusive transport, $J_{i}$ :

$$
N_{i}=v_{i} C_{i}+J_{i}, \quad i \in\{X, S, O, g O\} .
$$

The diffusive transport is modeled using Fick's law:

$$
J_{i}=-D_{i} \frac{\partial C_{i}}{\partial z}, \quad i \in\{X, S, O, g O\} .
$$

\section{F. Gas-liquid transport}

The transport of oxygen from the gas phase to the liquid phase is governed by the relation

$$
J_{g l, O}=\left(k_{L} a\right)_{O}\left(C_{s a t, O}-C_{O}\right),
$$

where Henry's law in combination with the ideal gas law provides the saturation concentration of oxygen, i.e.

$$
C_{\text {sat }, O}=\frac{P_{g O}}{H_{O}}=\frac{R T}{M_{w O} H_{O}} C_{g O} .
$$

$H_{O}$ is the Henry constant for oxygen, $P_{g O}$ is the partial pressure of oxygen in the gas phase, $R$ is the gas constant, $T$ is the temperature, $M_{w O}$ is the molar weight of oxygen, and $C_{g O}$ is the concentration of oxygen in the gas phase.

\section{G. Stoichiometry and kinetics}

The overall conversion of methanol $(S)$ to biomass $(X)$ is governed by the stoichiometric relation

$$
\begin{array}{r}
\mathrm{CH}_{3} \mathrm{OH}+Y_{S N} \mathrm{HNO}_{3}+Y_{S O} \mathrm{O}_{2} \rightarrow \\
Y_{S X} X+Y_{S C} \mathrm{CO}_{2}+Y_{S W} \mathrm{H}_{2} \mathrm{O}
\end{array}
$$

which can also be formulated as

$$
\begin{gathered}
Y_{X S} \mathrm{CH}_{3} \mathrm{OH}+Y_{X N} \mathrm{HNO}_{3}+Y_{X O} \mathrm{O}_{2} \rightarrow \\
X+Y_{X C} \mathrm{CO}_{2}+Y_{X W} \mathrm{H}_{2} \mathrm{O} .
\end{gathered}
$$

Table I reports the yield coefficients for this overall reaction. The stoichiometry implies that the production rate of substrate (methanol), $R_{S}$, and the production rate of oxygen in the liquid phase, $R_{O}$, are related to the production rate of biomass, $R_{X}$, by

$$
\begin{aligned}
R_{S}=-\gamma_{S} R_{X}, & \gamma_{S}=\frac{M_{w S}}{M_{w X} Y_{S X}}, \\
R_{O}=-\gamma_{O} R_{X}, & \gamma_{O}=\frac{M_{w O} Y_{S O}}{M_{w X} Y_{S X}} .
\end{aligned}
$$

TABLE I

YIELD COEFFICIENTS

\begin{tabular}{lccccc}
\hline & $i$ & $\begin{array}{c}Y_{S i} \\
{[\mathrm{~mol} / \mathrm{mol}]}\end{array}$ & $\begin{array}{c}Y_{X i} \\
{[\mathrm{~mol} / \mathrm{mol}]}\end{array}$ & $\begin{array}{c}M_{w i} \\
{[\mathrm{~g} / \mathrm{mol}]}\end{array}$ & $\begin{array}{c}W_{X i} \\
{[\mathrm{~g} / \mathrm{g}]}\end{array}$ \\
\hline $\mathrm{CH}_{3} \mathrm{OH}$ & $S$ & 1.000 & 1.366 & 32.042 & 1.778 \\
$\mathrm{HNO}_{3}$ & $N$ & 0.146 & 0.199 & 63.013 & 0.510 \\
$\mathrm{O}_{2}$ & $O$ & 0.439 & 0.600 & 31.999 & 0.779 \\
$\mathrm{CH}_{1.8} \mathrm{O}_{0.5} \mathrm{~N}_{0.2}$ & $X$ & 0.732 & 1.000 & 24.626 & 1.000 \\
$\mathrm{CO}_{2}$ & $C$ & 0.268 & 0.366 & 44.010 & 0.654 \\
$\mathrm{H}_{2} \mathrm{O}$ & $W$ & 1.415 & 1.933 & 18.015 & 1.414 \\
\hline
\end{tabular}

The production rate of biomass, $R_{X}$, is given by

$$
R_{X}=\mu\left(C_{S}, C_{O}\right) C_{X}
$$

where $\mu=\mu\left(C_{S}, C_{O}\right)$ is the specific growth rate. The specific growth rate of Methylococcus Capsulatus in a methanol medium is given by the Monod-Haldane expression

$$
\mu=\mu\left(C_{S}, C_{O}\right)=\mu_{\max } \mu_{S}\left(C_{S}\right) \mu_{O}\left(C_{O}\right),
$$

where the growth factors, $\mu_{S}\left(C_{S}\right)$ and $\mu_{O}\left(C_{O}\right)$, are

$$
\begin{aligned}
\mu_{S}\left(C_{S}\right) & =\frac{C_{S}}{K_{S}+C_{S}+C_{S}^{2} / K_{I}}, \\
\mu_{O}\left(C_{O}\right) & =\frac{C_{O}}{K_{O}+C_{O}} .
\end{aligned}
$$

\section{H. Stochastic effects and parameters}

In this paper, we model the variation of some key parameters as stochastic processes,

$$
\begin{aligned}
d \mu_{\max }(t) & =\kappa_{\mu}\left(\bar{\mu}_{\max }-\mu_{\max }(t)\right) d t+\sigma_{\mu} d w_{\mu}(t), \\
d \gamma_{S}(t) & =\kappa_{\gamma_{S}}\left(\bar{\gamma}_{S}-\gamma_{S}(t)\right) d t+\sigma_{\gamma_{S}} d w_{\gamma_{S}}(t), \\
d \gamma_{O}(t) & =\kappa_{\gamma_{O}}\left(\bar{\gamma}_{O}-\gamma_{O}(t)\right) d t+\sigma_{\gamma_{O}} d w_{\gamma_{O}}(t),
\end{aligned}
$$

around the mean (i.e. the nominal) values of the parameters. Similarly, we consider stochastic variations of the feed substrate concentration according to

$d C_{F, S}(t)=\kappa_{C_{F, S}}\left(\bar{C}_{F, S}(t)-C_{F, S}(t)\right) d t+\sigma_{C_{F, S}} d w_{C_{F, S}}(t)$.

\section{NONLINEAR STATE ESTIMATION}

Using spatial discretization on $N_{z}$ equidistantly spaced finite volumes, the mathematical model for the U-loop reactor presented in Section II can be compactly represented as

$$
d \boldsymbol{x}_{d}(t)=f\left(\boldsymbol{x}_{d}(t), \boldsymbol{x}_{s}(t), u(t), \theta\right) d t
$$

and the corresponding compact model for the stochastic effects described in Section II-H is

$$
d \boldsymbol{x}_{s}(t)=f_{s}\left(\boldsymbol{x}_{s}(t), u(t), \theta\right) d t+\sigma_{s} d \boldsymbol{w}_{s}(t) .
$$

In this section, we describe the EKF for continuousdiscrete-time stochastic systems in the form

$$
\begin{aligned}
d \boldsymbol{x}(t) & =f(\boldsymbol{x}(t), u(t), \theta) d t+\sigma(\boldsymbol{x}(t), u(t), \theta) d \boldsymbol{w}(t), \\
\boldsymbol{z}(t) & =g(\boldsymbol{x}(t), \theta), \\
\boldsymbol{y}\left(t_{k}\right) & =\boldsymbol{z}\left(t_{k}\right)+\boldsymbol{v}\left(t_{k} ; \theta\right) .
\end{aligned}
$$


The combined model (21) is a special case of the stochastic differential equation (22a), (22b) is a model of the process outputs, and (22c) is a model of the measurements. $\boldsymbol{x}$ is the state vector, $u$ is the vector of manipulated inputs, $\theta$ is the parameter vector, $\boldsymbol{z}$ is the vector of outputs, and $\boldsymbol{y}$ are measurements of the outputs (at discrete times, $t_{k}$ ) which are corrupted by measurement noise, $\boldsymbol{v}\left(t_{k} ; \theta\right) \sim$ $N_{i i d}\left(0, R_{v}(\theta)\right) . \boldsymbol{w}(t)$ is a standard Wiener process, i.e. $d \boldsymbol{w}(t) \sim N_{i i d}(0, I d t)$. The initial states are normally distributed: $\boldsymbol{x}\left(t_{0}\right) \sim N\left(\bar{x}_{0}, P_{0}\right)$.

\section{A. Simulation and temporal discretization}

We use a semi-implicit method [22] to discretize the stochastic differential equation (22a):

$$
\begin{aligned}
x_{k, n+1}-x_{k, n}=f & \left(x_{k, n+1}, u_{k}, \theta\right) \Delta t_{k, n} \\
& +\sigma\left(x_{k, n}, u_{k}, \theta\right) \Delta w_{k, n} .
\end{aligned}
$$

$\Delta w_{k, n}$ is a realization of $\Delta \boldsymbol{w}_{k, n} \sim N_{i i d}\left(0, I \Delta t_{k, n}\right)$. The states, $x_{k, n+1}$, are obtained by solution of

$$
\begin{aligned}
R_{k, n}= & R_{k, n}\left(x_{k, n+1}\right) \\
= & x_{k, n+1}-f\left(x_{k, n+1}, u_{k}, \theta\right) \Delta t_{k, n} \\
& \quad-x_{k, n}-\sigma\left(x_{k, n}, u_{k}, \theta\right) \Delta w_{k, n} \\
& =0, \quad
\end{aligned}
$$

using a variant of Newton-Raphson's method.

\section{B. Extended Kalman filter}

The EKF for the continuous-discrete-time stochastic system (22) consists of a filter and a one-step predictor.

1) Initialization: The initialization of the EKF is based on the prior information represented by the distribution of the initial states. Consequently, $\hat{x}_{0 \mid-1}=\bar{x}_{0}$ and $P_{0 \mid-1}=P_{0}$.

2) Filter step: At each discrete time $k$, i.e. time $t_{k}$, the filter assumes that $\hat{x}_{k \mid k-1}$ and $P_{k \mid k-1}$ are available. It computes the one-step prediction of the output, $\hat{z}_{k \mid k-1}$, and the measurement, $\hat{y}_{k \mid k-1}$, by

$$
\hat{y}_{k \mid k-1}=\hat{z}_{k \mid k-1}=g\left(\hat{x}_{k \mid k-1}, \theta\right) .
$$

Linearization of the output equation provides

$$
C_{k}=\frac{\partial g}{\partial x}\left(\hat{x}_{k \mid k-1}, \theta\right)
$$

such that the innovation, $e_{k}$, and its covariance, $R_{e, k}=$ $\left\langle\boldsymbol{y}_{k \mid k-1}, \boldsymbol{y}_{k \mid k-1}\right\rangle$, may be computed by

$$
\begin{aligned}
e_{k} & =y_{k}-\hat{y}_{k \mid k-1}, \\
R_{e, k} & =C_{k} P_{k \mid k-1} C_{k}^{\prime}+R_{v}(\theta),
\end{aligned}
$$

where $y_{k}$ is the measurement. The Kalman filter gain, $K_{f x, k}=\left\langle\boldsymbol{x}_{k \mid k-1}, \boldsymbol{y}_{k \mid k-1}\right\rangle\left\langle\boldsymbol{y}_{k \mid k-1}, \boldsymbol{y}_{k \mid k-1}\right\rangle^{-1}$, is

$$
K_{f x, k}=P_{k \mid k-1} C_{k}^{\prime} R_{e, k}^{-1} \text {. }
$$

The Kalman filter gain, $K_{f x, k}$, is used in the computation of the filtered state, $\hat{x}_{k \mid k}$, and its covariance, $P_{k \mid k}=\left\langle\boldsymbol{x}_{k \mid k-1}, \boldsymbol{x}_{k \mid k-1}\right\rangle-$ $\left\langle\boldsymbol{x}_{k \mid k-1}, \boldsymbol{y}_{k \mid k-1}\right\rangle\left\langle\boldsymbol{y}_{k \mid k-1}, \boldsymbol{y}_{k \mid k-1}\right\rangle^{-1}\left\langle\boldsymbol{y}_{k \mid k-1}, \boldsymbol{x}_{k \mid k-1}\right\rangle$. They are computed according to

$$
\begin{aligned}
& \hat{x}_{k \mid k}=\hat{x}_{k \mid k-1}+K_{f x, k} e_{k}, \\
& P_{k \mid k}=P_{k \mid k-1}-K_{f x, k} R_{e, k} K_{f x, k}^{\prime} .
\end{aligned}
$$

3) One-step predictor: The one-step prediction of the states are computed by solution of the initial value problem

$$
\frac{d}{d t} \hat{x}_{k}(t)=f\left(\hat{x}_{k}(t), u_{k}, \theta\right), \quad \hat{x}_{k}\left(t_{k}\right)=\hat{x}_{k \mid k} .
$$

In order to compute the one-step prediction of the covariance, we compute the sensitivities of the one-step prediction of the states at time $t$ with respect to the states at time $s, \Phi_{k}(t, s)$, by solving the initial value problem

$$
\frac{d}{d t} \Phi_{k}(t, s)=A_{k}(t) \Phi_{k}(t, s), \quad \Phi_{k}(s, s)=I,
$$

where

$$
A_{k}(t)=\frac{\partial f}{\partial x}\left(\hat{x}_{k}(t), u_{k}, \theta\right) .
$$

Using these sensitivities, we compute the covariance at time $t$ by [23]

$$
\begin{aligned}
P_{k}(t)=\Phi_{k}( & \left(t, t_{k}\right) P_{k \mid k} \Phi_{k}\left(t, t_{k}\right)^{\prime} \\
& +\int_{t_{k}}^{t} \Phi_{k}(t, s) \sigma_{k}(s) \sigma_{k}(s)^{\prime} \Phi_{k}(t, s)^{\prime} d s,
\end{aligned}
$$

where

$$
\sigma_{k}(t)=\sigma\left(\hat{x}_{k}(t), u_{k}, \theta\right)
$$

We use Euler's implicit method (with multiple time steps in between measurements) together with a variant of NewtonRaphson's method to solve the initial value problems (30) and (31), and we use a left rectangle rule to approximate the integral in (33).

\section{NUMERICAL EXAMPLE}

In this section, we present a numerical example of state estimation in the U-loop reactor during startup based on the economic optimizing control strategy presented by Drejer et al. [19] which is stabilized by a P-controller (i.e. using feedback). The P-controller changes the substrate feed flow rate every $15 \mathrm{~s}$ based on the substrate concentration in the top tank. The feedback dynamics are not represented in the dynamical model used by the EKF. Furthermore, the EKF uses average values of the substrate feed flow rate computed by the P-controller (averaged over each sampling interval). We consider a time interval of $30 \mathrm{~h}$, and the EKF uses measurements of the concentration of the dissolved oxygen at positions $z=4 \mathrm{~m}, z=7 \mathrm{~m}$, and $z=10 \mathrm{~m}$ in the U-loop pipe. These measurements are obtained every third minute. We discretize the U-loop pipe using 20 equidistant finite volumes. Consequently, we measure the concentrations of the dissolved oxygen in the 7'th, 12'th, and 17'th finite volumes in the discretized U-loop pipe. The resulting dynamical system contains 83 state variables as well as the 3 uncertain parameters, $\mu_{\max }, \gamma_{S}$, and $\gamma_{O}$, and the uncertain substrate feed concentration, $C_{F, S}$, as described in Section 
II-H. Consequently, the EKF estimates the 87 state variables in the combined dynamical system.

We use a stochastic simulation to represent the true U-loop reactor and to compute the measurements of the dissolved oxygen concentrations. In this stochastic simulation, we use 12 time steps in between the measurements corresponding to the update frequency of the P-controller. We use 6 time steps between the measurements in the EKF. We provide more detail on this numerical example in the Appendix.

Fig. 2 shows the manipulated inputs, and Fig. 3 shows the filtered estimates of the concentrations of biomass, substrate, and dissolved oxygen in the top tank computed using the EKF (in blue) together with the true concentrations (in green). Furthermore, it shows the absolute deviations of the filtered estimates from the true concentrations. The EKF is able to accurately estimate the concentrations of biomass and dissolved oxygen in the top tank. However, the estimates of the substrate concentration in the top tank deviate significantly from the true values. This is possibly because the EKF does not estimate the substrate concentration in the feed, $C_{F, S}$, accurately during the first $15 \mathrm{~h}$ of the startup. The filtered estimate of $C_{F, S}$ is shown in Fig. 4 together with the filtered estimates of the uncertain model parameters $\mu_{\max }$, $\gamma_{S}$, and $\gamma_{O}$ (in blue) as well as the true values (in green) and the nominal values (in black) of these parameters.

In the implementation of the EKF, the evaluation of the right-hand side function $f(x(t), u(t), \theta)$ in the combined dynamical system (22) is implemented in C, and the rest is implemented in Matlab R2016b. The average computation times of the filtering step and the one-step predictor are $0.1 \mathrm{~ms}$ and $9.4 \mathrm{~ms}$ which are negligible compared to the sampling time.

\section{CONCLusion}

In this work, we describe the EKF for nonlinear state estimation in a U-loop reactor for SCP production. The EKF requires a dynamical model of the $\mathrm{U}$-loop reactor. We present such a dynamical model which consists of both stochastic partial differential equations and stochastic differential equations, and we use a finite-volume approach to spatially discretize the stochastic partial differential equations. Furthermore, we demonstrate, using a numerical example, that the EKF can be used to estimate the state of the U-loop reactor during startup. In this example, we use a previously developed economic optimizing control strategy together with a P-controller for stabilization. The EKF is implemented using Matlab and $\mathrm{C}$, and the computation time is negligible compared to the sampling time indicating that real-time implementation is feasible. Future work could involve using the UKF for improving the accuracy of the state estimation in the U-loop reactor.

\section{REFERENCES}

[1] A. H. Jazwinski, Stochastic Processes and Filtering Theory. San Diego, CA, USA: Academic Press, 1970.

[2] T. Kailath, A. H. Sayed, and B. Hassibi, Linear Estimation. Prentice Hall, 2000

[3] A. Gelb, Applied Optimal Estimation. MIT Press, 1974.
[4] D. Simon, Optimal State Estimation. Kalman, $H_{\infty}$, and Nonlinear Approaches. Wiley, 2006.

[5] A. M. Fraser, Hidden Markov Models and Dynamical Systems. SIAM, 2008.

[6] J. B. Rawlings, D. Q. Mayne, and M. M. Diehl, Model Predictive Control: Theory, Computation, and Design, 2nd ed. Madison, WI, USA: Nob Hill Publishing, 2017.

[7] S. Gillijns, O. B. Mendoza, J. Chandrasekar, B. L. R. De Moor, D. S. Bernstein, and A. Ridley, "What is the ensemble Kalman filter and how well does it work?" in Proceedings of the 2006 American Control Conference, Minneapolis, Minnesota, USA, 2006, pp. 4448-4453.

[8] M. Nørgaard, N. K. Poulsen, and O. Ravn, "New developments in state estimation for nonlinear systems," Automatica, vol. 36, pp. 1627-1638, 2000

[9] T. S. Schei, "A finite-difference method for linearization in nonlinear estimation algorithms," Automatica, vol. 33, no. 11, pp. 2053-2058, 1997.

[10] D. Dochain, "State and parameter estimation in chemical and biochemical processes: a tutorial," Journal of Process Control, vol. 13, pp. 801-818, 2003.

[11] J. B. Rawlings and B. R. Bakshi, "Particle filtering and moving horizon estimation," Computers and Chemical Engineering, vol. 30, pp. 1529$1541,2006$.

[12] T. S. Schei, "On-line estimation for process control and optimization applications," Journal of Process Control, vol. 18, pp. 821-828, 2008.

[13] S. Kolås, B. A. Foss, and T. S. Schei, "Noise modeling concepts in nonlinear state estimation," Journal of Process Control, vol. 19, pp. $1111-1125,2009$

[14] D. F. Olsen, J. B. Jørgensen, J. Villadsen, and S. B. Jørgensen, "Modeling and simulation of single cell protein production," IFAC Proceedings Volumes, vol. 43, no. 6, pp. 502-507, 2010.

[15] O. A. Prado-Rubio, J. B. Jørgensen, and S. B. Jørgensen, "Systematic model analysis for single cell protein (SCP) production in a U-loop reactor," Computer Aided Chemical Engineering, vol. 28, pp. 319-324, 2010.

[16] A. M. Al Taweel, Q. Shah, and B. Aufderheide, "Effect of mixing on microorganism growth in loop bioreactors," International Journal of Chemical Engineering, no. Article ID 984827, 2012.

[17] M. Wu, J. K. Huusom, K. V. Gernaey, and U. Krühne, "Modelling and simulation of a U-loop reactor for single cell protein production," Computer Aided Chemical Engineering, vol. 38, pp. 1287-1292, 2016.

[18] L. A. H. Petersen, J. Villadsen, S. B. Jørgensen, and K. V. Gernaey, "Mixing and mass transfer in a pilot scale U-loop bioreactor," Biotechnology and Bioengineering, vol. 114, no. 2, pp. 344-354, 2017.

[19] A. Drejer, T. Ritschel, S. B. Jørgensen, and J. B. Jørgensen, "Economic optimizing control for single-cell protein production in a U-loop reactor," Computer Aided Chemical Engineering, vol. 40, pp. 17591764, 2017.

[20] L. N. Petersen and J. B. Jørgensen, "Real-time economic optimization for a fermentation process using model predictive control," in European Control Conference (ECC) 2014. IEEE, 2014, pp. 1831-1836.

[21] D. F. Olsen, J. B. Jørgensen, J. Villadsen, and S. B. Jørgensen, "Optimal operating points for SCP production in the U-loop reactor," IFAC Proceedings Volumes, vol. 43, no. 5, pp. 499-504, 2010.

[22] K. Burrage and T. Tian, "The composite Euler method for stiff stochastic differential equations," Journal of Computational and Applied Mathematics, vol. 131, pp. 407-426, 2001.

[23] J. B. Jørgensen, M. R. Kristensen, P. G. Thomsen, and H. Madsen, "New extended Kalman filter algorithms for stochastic differential algebraic equations," in Assessment and Future Directions of Nonlinear Model Predictive Control, ser. Lecture Notes in Control and Information Sciences. Springer-Verlag Berlin Heidelberg, 2007, vol 358, pp. 359-366.

\section{APPENDIX}

Table II shows parameters used in the numerical example. The units of the diffusion coefficients, $\sigma_{\mu}, \sigma_{\gamma_{S}}, \sigma_{\gamma_{O}}$, and $\sigma_{C_{F, S}}$, contain a factor of $1 / \mathrm{h}^{1 / 2}$ because the unit of the increment of the Wiener process, $d \boldsymbol{w}(t)$, in the stochastic continuous-discrete-time system (22) is $\mathrm{h}^{1 / 2}$, i.e. the unit of the covariance of this increment is $h$. 

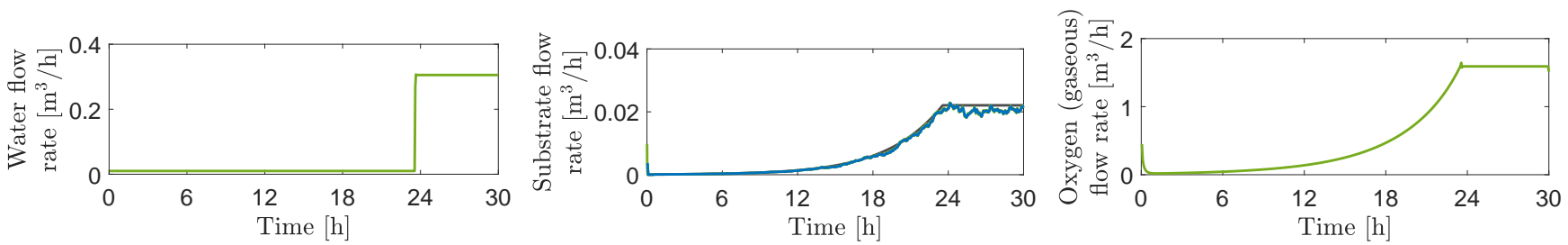

Fig. 2. Manipulated inputs: feed flow rates of water, substrate, and gaseous oxygen. For the substrate flow rate, the green curve represents the values used in the stochastic simulation of the U-loop reactor, the blue curve represents the values used in the EKF, and the black curve represents the open-loop economically optimal strategy computed by Drejer et al. [19] (the green and blue curves almost completely coincide). For the water and oxygen flow rates, the green curve is used both in the stochastic simulation and in the EKF.
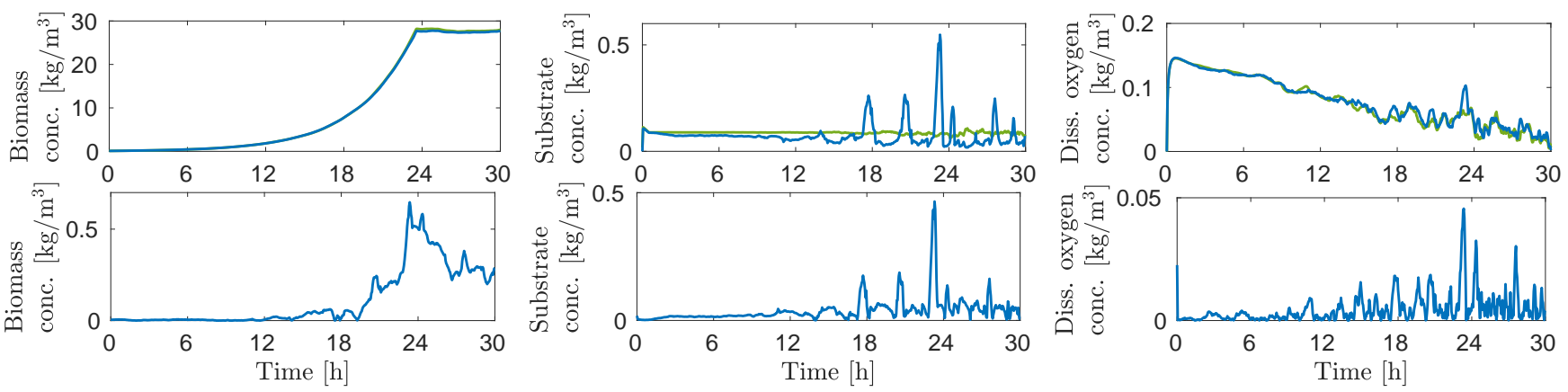

Fig. 3. Top row: filtered estimates of the concentrations in top tank of the U-loop reactor obtained with the EKF (blue) together with the true concentrations (green). Bottom row: absolute differences between the filtered estimates and the true values of these concentrations.
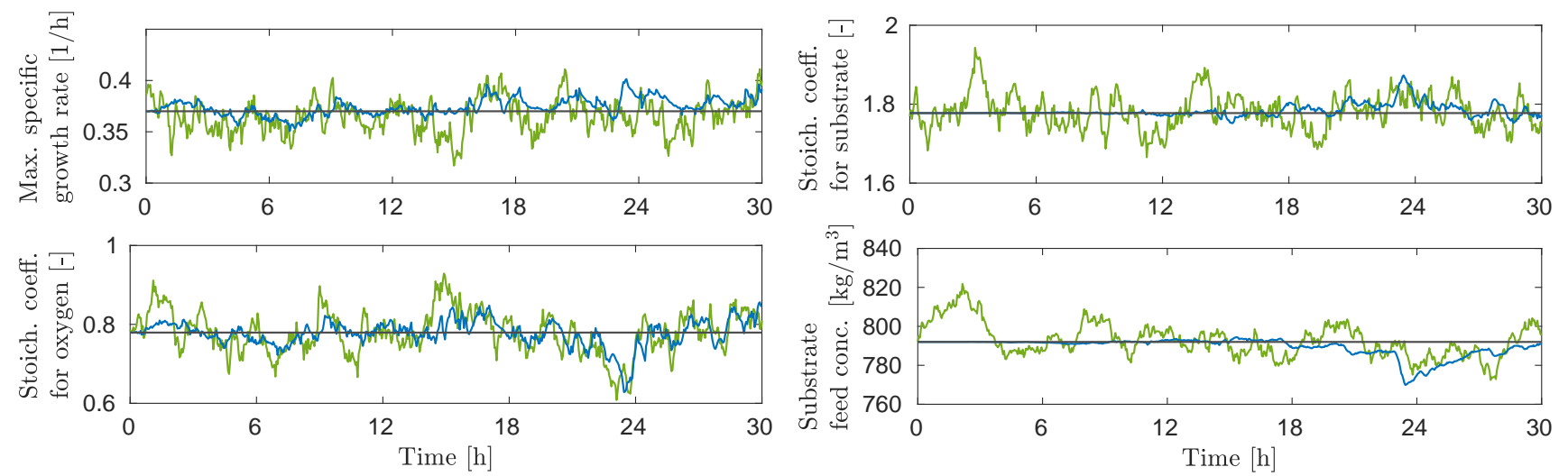

Fig. 4. Filtered estimates of the maximum specific growth rate, $\mu_{\max }$, the stoichiometric coefficients for substrate and oxygen, $\gamma_{S}$ and $\gamma_{O}$, and the substrate concentration in the inlet, $C_{F, S}$ computed using the EKF (blue) together with the true (green) and nominal (black) values of these parameters.

The mean of the initial states, $\bar{x}_{0}$, is constructed such that the biomass concentration in the U-loop pipe and the top tank are $0.1 \mathrm{~kg} / \mathrm{m}^{3}$, and the reactor contains no substrate or oxygen (neither dissolved or in gaseous form). The covariance of the initial states, $P_{0}$, used in the EKF and to sample the true initial states is a diagonal matrix whose entries are $10^{-4}$ (the units vary).

The P-controller used to stabilize the open-loop economic optimizing control strategy presented by Drejer et al. [19] is

$$
\tilde{F}_{S}=F_{S}^{*}+K_{c S}\left(\bar{C}_{S}^{*}-\bar{C}_{S}\right)
$$

where $F_{S}^{*}$ and $\bar{C}_{S}^{*}$ are the optimized open-loop substrate feed flow rate and the substrate concentration in the top tank. $\bar{C}_{S}$ is the actual substrate concentration in the top tank, and in this example, we use $K_{c S}=0.1$.
TABLE II

PARAMETER VALUES USED IN THE NUMERICAL EXAMPLE.

\begin{tabular}{lrr} 
Symbol & Value & Unit \\
\hline $\bar{\mu}_{\max }$ & 0.37 & $1 / \mathrm{h}$ \\
$\bar{\gamma}_{S}$ & 1.78 & - \\
$\bar{\gamma}_{O}$ & 0.78 & - \\
$\bar{C}_{F, S}$ & 792 & $\mathrm{~kg} / \mathrm{m}^{3}$ \\
$\kappa_{\mu}$ & 2 & $1 / \mathrm{h}$ \\
$\kappa_{\gamma_{S}}$ & 2 & $1 / \mathrm{h}$ \\
$\kappa_{\gamma_{O}}$ & 2 & $1 / \mathrm{h}$ \\
$\kappa_{C_{F, S}}$ & 0.5 & $1 / \mathrm{h}$ \\
$\sigma_{\mu}$ & 0.04 & $1 / \mathrm{h}^{3 / 2}$ \\
$\sigma_{\gamma_{S}}$ & 0.1 & $1 / \mathrm{h}^{1 / 2}$ \\
$\sigma_{\gamma_{O}}$ & 0.1 & $1 / \mathrm{h}^{1 / 2}$ \\
$\sigma_{C_{F, S}}$ & 10.0 & $\mathrm{~kg} /\left(\mathrm{m}^{3} \mathrm{~h}^{1 / 2}\right)$ \\
$R_{v}$ & $10^{-4} \cdot \mathrm{I}_{3 \times 3}$ & $\left(\mathrm{~kg} / \mathrm{m}^{3}\right)^{2}$
\end{tabular}

